\title{
Guías de práctica clínica basadas en la evidencia
}

\author{
Carlos Manterola D. ${ }^{1,2,3}$, Tamara Otzen H. ${ }^{2,3}$, Nayely García ${ }^{3}$ y Miriam Mora V..$^{3,4}$
}

'Departamento de Cirugía Universidad de La Frontera. Temuco, Chile.

${ }^{2}$ Centro de Estudios

Morfológicos y Quirúrgicos

(CEMyQ), Universidad de La

Frontera. Temuco, Chile.

3Programa de Doctorado en Ciencias Médicas, Universidad de La Frontera. Temuco, Chile.

${ }^{4}$ Universidad del Azuay,

Cuenca, Ecuador.

Recibido el 21 de diciembre

de 2018 y aceptado para

publicación el 4 de abril de

Correspondencia a:

Dr. Carlos Manterola D. carlos.manterola@ufrontera.cl

\section{Evidence-based clinical practice guidelines}

Clinical Practice Guidelines (CPG) are useful tools to organize the best available scientific evidence, and to collaborate with clinicians in health decision making. The aim of this article was to describe concepts that allow understanding the importance of CPGs in daily clinical practice. CPGs arise from a series of steps that are carried out with varying intensity depending on the subject matter, among which are: methodological aspects, systematic review of the literature, publication and implementation, periodic review, and update. Among the advantages of CPGs is the improvement in health outcomes because its use helps to promote adequate health decision-making, reducing undue variability in the choice of treatments. On the other hand, PCs are very useful instruments for those who must plan and manage health costs, since they allow improving the efficiency of resources, and control health expenses while maintaining the quality of care. Among the disadvantages, it is described that eventually do not achieve to change a behavior, unless they are accompanied by active strategies such as reminder, audit and feedback systems, etc.; that physicians are resistant to behavioral changes, put attitude barriers in accepting the validity of recommendations, etc.

Key words: "Practice guidelines as topic"; "Practice guideline" [Publication Type]; "Evidence-based medicine".

\section{Resumen}

Las Guías de Práctica Clínica (GPC), son instrumentos adecuados para sistematizar la mejor evidencia científica disponible para colaborar con los clínicos en la toma de decisiones sanitarias. El objetivo de este artículo fue describir conceptos que permitan comprender la importancia de la utilización de las GPC en la práctica clínica cotidiana. En general, las GPC surgen de una serie de pasos que requieren distintos procedimientos dependiendo de la temática, entre los que destacan los aspectos metodológicos, la revisión sistemática de la literatura, la publicación e implementación, y la revisión y actualización periódica. Entre las ventajas de contar con GPC destaca el hecho de mejorar los resultados en salud debido a que su uso ayuda a promover acciones sanitarias adecuadas, reduciendo la variabilidad injustificada en la elección de tratamientos. Por otra parte, las GPC son instrumentos de gran utilidad para quienes deben planificar y gestionar gastos en salud, debido a que permiten mejorar la eficiencia de los recursos y controlar los costes sanitarios manteniendo la calidad asistencial. Entre las desventajas, se describen que con cierta frecuencia (y a pesar de todo) no logran cambiar una conducta, a menos que se acompañen de estrategias activas como sistemas de recordatorio, auditoria y retroalimentación, etc.; en parte, porque los profesionales de salud son resistentes a los cambios de comportamiento, ponen barreras de actitud en la aceptación de la validez de las recomendaciones, etc.

Palabras clave: guías de práctica clínica; medicina basada en la evidencia.

\section{Introducción}

Las Guías de Práctica Clínica (GPC), constituyen un instrumento significativo en la estrategia de implementación del modelo de atención de salud. Componen un conjunto de recomendaciones desa- rrolladas de forma sistemática y basadas en la mejor evidencia científica disponible con el propósito de facilitar al equipo de salud y a las personas, la toma de decisiones sobre su asistencia sanitaria ${ }^{1}$.

Se podrían definir de una forma simple, como herramientas útiles para organizar la mejor eviden- 
cia científica disponible para la toma de decisiones clínicas o en salud; como normas, criterios o directrices desarrollados de forma sistemática, para colaborar con los clínicos en la toma de decisiones sanitarias respecto del cuidado de la salud en escenarios clínicos específicos ${ }^{2}$.

Por otra parte, se podría decir que ya sean formales o informales, las guías clínicas constituyen las bases de toda práctica clínica, debido a que representan los fundamentos para la toma de decisiones clínicas, son relevantes en el ámbito docente, encarnan ayudas de memoria para problemas comunes o complejos; son de utilidad para la valoración de patrones y estándares de atención ${ }^{3}$.

$\mathrm{Su}$ beneficio es indiscutible en casos de problemas sanitarios frecuentes o de alto costo; cuando existe una gran variabilidad de conductas en la práctica clínica; y cuando existe considerable información científica sobre la prestación en cuestión (en ocasiones incluso discordante $)^{4}$.

El propósito principal de una GPC es asegurar que al tratar los pacientes de acuerdo a ellas se obtendrán los resultados sanitarios deseados ${ }^{5}$.

El objetivo de este artículo fue describir algunos conceptos que permitan comprender la importancia de la utilización de las GPC en la práctica clínica cotidiana.

\section{GPC en el quehacer cotidiano del clínico}

La práctica clínica es un acto rutinario, pero que lleva implícito una variabilidad no sólo relacionada con el sujeto que está siendo atendido, sino que también por los profesionales sanitarios que lo atienden. Es así como no es inusual que clínicos de un mismo centro sanitario diagnostiquen y traten a un paciente de similares condiciones de forma diferente, o incluso al mismo individuo si éste consulta una "segunda opinión". Esta situación puede llegar a extremos tales, que puede impedir la comparación y evaluación de resultados entre clínicos, grupos de trabajo y centros sanitarios ${ }^{6}$.

Si bien es cierto que los pacientes pueden ser distintos en una serie de parámetros y que una misma entidad nosológica puede presentarse con manifestaciones clínicas diversas; se cuenta con herramientas desarrolladas a partir de la práctica clínica basada en la evidencia, que ayudan a establecer ciertos lineamientos que permiten orientar a la toma de decisiones en salud, lo que permite que la variabilidad deje de ser un problema permanente ${ }^{7}$.

Con esta filosofía nacen las GPC, instrumentos diseñados con el objetivo de uniformar el actuar del profesional sanitario, así como de mejorar la calidad de las prestaciones en salud ${ }^{5}$.

Sin embargo, y lamentablemente, existe una tendencia generalizada a crear GPC sin consideración alguna respecto de algunas cuestiones metodológicas inherentes a la información que avala la nueva tecnología sanitaria que se aplicará, la opinión de quienes tendrán que aplicarlas, los usuarios de éstas, los costes involucrados en la aplicación, el beneficio esperado de ellas, etc ${ }^{8}$. Una GPC debe ser validable, aplicable, flexible, tener claridad de criterios, realismo y un carácter multidisciplinario.

Existen organismos que se dedican a elaborar GPC (Tabla 1); así como hay otros que se dedican a compilar (búsqueda y almacenamiento) GPC (Tabla 2).

\section{Tipos de GPC existentes}

Antes de explicar los distintos tipos existentes de GPC, nos parece conveniente aclarar algunos conceptos relacionados, que a veces se confunden con una GPC. Nos referimos a los protocolos de atención y a las vías clínicas 9 . Los primeros, corresponden a documentos que describen de forma resumida un conjunto de procedimientos necesarios para el cuidado de un escenario sanitario específico. Pueden formar parte de una GPC y se emplean

Tabla 1. Centros que elaboran GPC

\begin{tabular}{|lll|}
\hline Centro & País & Dirección electrónica \\
NHMRC & Australia & $\begin{array}{l}\text { http://www.health.gov.au/nhmrc/publications/cphome. } \\
\text { html }\end{array}$ \\
CTFPHC & Canadá & http://www.ctfphc.org \\
SIGN & Escocia & http://www.sign.ac.uk \\
SIGNet & Escocia & http://www.rcgp-signet.co.uk \\
USPSTF & EE. UU. & https://www.uspreventiveservicestaskforce.org \\
HSTAT & EE. UU. & http://text.nlm.nih.gov \\
AAP & EE. UU. & http://www.aap.org/policy/paramtoc.html \\
AHRQ & EE. UU. & http://text.nlm.nih.gov \\
NICE & Inglaterra & http://www.nice.org.uk \\
NZGG & N. Zelanda & http://www.nzgg.org.nz \\
\hline
\end{tabular}

NHMRC: National Health and Medical Research; CTFPHC: Canadian Task Force on Preventive Health Care; SIGN: Scottish Intercollegiate Guidelines Network; SIGNet: Scottish Intercollegiate Guidelines Network (orientada a atención primaria); USPSTF: US Preventive Service Task Force; HSTAT: Health Services Technology Assessment Texts; AAP: American Academy of Pediatrics; AHRQ: Clinical Practice Guidelines; NICE: National Institute for Clinical Excellence; NZGG: New Zealand Guidelines Group. 
ARTíCULO DE REVISIÓN

Tabla 2. Organismos compiladores (búsqueda y almacenamiento) DE GPC

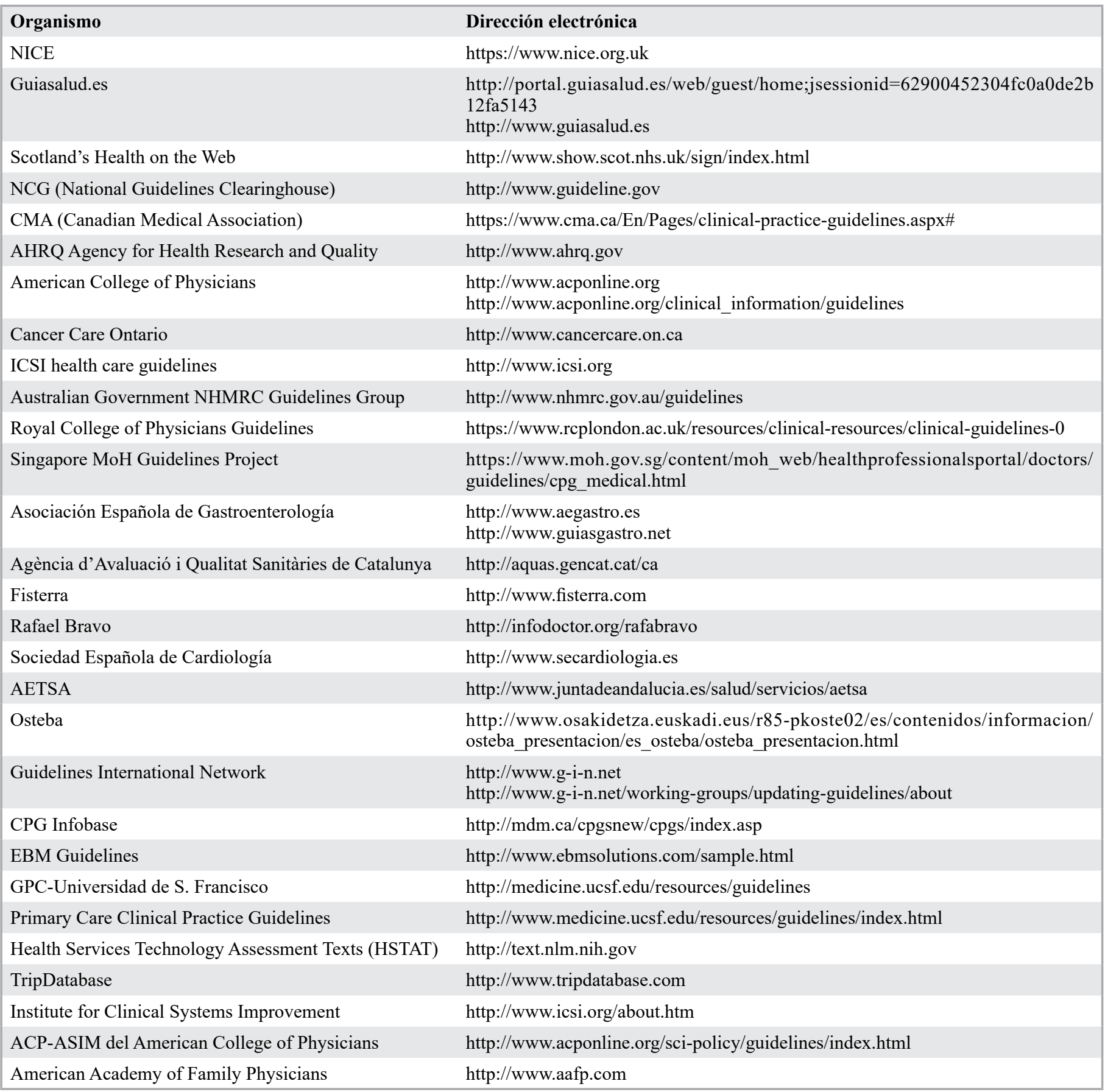

principalmente en situaciones críticas que requieren apego total a lo señalado (ej. urgencias). Por su parte las segundas, constituyen planes asistenciales que se destinan a pacientes con una patología de curso clínico predecible (también se les denominan guías prácticas, vías de atención, programas de atención en colaboración, etc.). Dicho de otra forma, representan una forma de adaptar una GPC al ejercicio habitual de un centro (versión operativa de una GPC). 
Existen GPC según contexto clínico, social y sanitario, metodología de elaboración, formas de difusión y procedimiento utilizado para su desarrollo ${ }^{1,5}$. 1. Basadas en opinión de expertos. Estas, se caracterizan porque no existe sistematización en su construcción (no estructuradas), y no hay una base en la evidencia científica (informales); razón por la que tienen un potencial de sesgos y de conflictos de interés considerable. Son de bajos costos y rápidas de construir. Sin embargo, la condición de experto se define según los contenidos de la GPC y pueden por ende, producirse desavenencias entre expertos. De hecho, hay algunas GPC que han sido confeccionadas por un solo experto (autor único). Por ejemplo, la puesta al día en resecciones hepáticas laparoscópicas propuesta por la Asociación Francesa de Cirugía Hépato-Bilio-Pancreática ${ }^{10}$. $\mathrm{O}$, como la puesta al día de las guías para el tratamiento de la colangitis aguda y la colecistitis del Comité de Revisión de Guías de Tokio ${ }^{11}$.

2. Basadas en consensos. Son formales. Se trabajan en base a "panel de expertos". Se elaboran con metodología estructurada y con cierta orientación en la práctica clínica basada en la evidencia (aunque suelen trabajar con evidencia implícita); por ende, tienen un riesgo de sesgos en la selección de los estudios utilizados para la confección de la GPC. Habitualmente involucra muchos participantes, lo que involucra diversos puntos de vista; y por ende, suelen ser lentas y costosas. Como el consenso es la meta, se puede llegar a él a expensas de la evidencia; sin descontar los potenciales enfrentamientos entre participantes. Por ejemplo, el consenso de la Sociedad ERAS para gastrectomía $^{12}$; o el consenso Asia-Pacífico de recomendaciones para el tratamiento endoscópico e intervencional del colangiocarcinoma hilar ${ }^{13}$; o las GPC para el diagnóstico y tratamiento del cáncer primitivo del hígado ${ }^{14}$.

3. Basadas en la evidencia. Son formales y estructuradas. Se identifican por la composición del panel de expertos que las construye (ej. centros especializados); la rigurosidad de la sistematización de individualización, búsqueda y valoración crítica de los artículos considerados (método explícito y reproducible); y por una definición del nivel de evidencia que apoya la recomendación por cada apartado, lo que les da una validez interna adecuada. Toda la metodología utilizada está disponible para ser revisada, lo que le otorga transparencia absoluta. Son lentas y caras ${ }^{7}$. Por ejemplo, la GPC basada en la evidencia para cáncer pancreático de la Sociedad Japonesa de páncreas $^{15}$.
Todas éstas, deben observar algunos principios fundamentales como: Estar construidas de tal manera, que permitan la reflexión del clínico ante una condición específica (flexibles y adaptables en el momento de su aplicación) y que se consideren los recursos con los que cuentan los servicios de salud que la implementarán. Por último, se ha de identificar las necesidades de capacitación técnica de los usuarios de esta GPC . $^{3}$.

Entre los propósitos del uso de GPC, se han de considerar los siguientes: Reducir o evitar conductas inapropiadas en la práctica clínica; disminuir la variabilidad en la práctica clínica; reducir la variabilidad en el acceso a determinadas intervenciones o tratamientos específicos; determinar el nivel asistencial y los mecanismos de derivación entre los diferentes niveles de atención; optimizar la comunicación con el paciente; servir como un instrumento de educación continua para el profesional de salud y mejorar la eficiencia del uso de los recursos ${ }^{1,16}$.

En la construcción de una GPC, se deben tomar en cuenta los siguientes hechos: Inspirarse en la mejor evidencia científica disponible (RS y EC); considerar al paciente en forma integral y a los profesionales implicados en la atención del paciente como elementos de un equipo de trabajo; el grupo que elabore una GPC debe ser multidisciplinario, incluyendo profesionales de todos los niveles de atención, con un trabajo en equipo y comunicación adecuada, con una apropiada capacitación del grupo que trabaja en la elaboración de la GPC y en los profesionales que la van a implementar. Finalmente, se ha de definir la técnica por consenso ${ }^{6}$.

Por otra parte, una GPC debe contar con ciertos atributos, como: Claridad en su lenguaje, de modo tal que su presentación tenga un orden lógico y fácil de seguir. Específica, respecto de sus alcances y propósitos. Flexible, independiente del tipo de escenario clínico (tratamiento, prevención, diagnóstico, etc.), de forma tal que se reflejen todas las alternativas de respuesta razonables y posibles. Aplicabilidad para poder llevarse a la práctica en cualquier lugar o circunstancia clínica. Validez, basada en que la información a partir de la que se construyó la GPC, es producto de una revisión bibliográfica rigurosa. Y, con actualizaciones periódicas, de forma tal de considerar las novedades que respecto del tema se van produciendo con el paso del tiempo ${ }^{2}$.

De esta forma, se puede concluir que las etapas más relevantes de la construcción de una GPC son los aspectos metodológicos, la revisión sistemática de la literatura, la publicación e implementación, y la revisión y actualización periódica ${ }^{3,6}$ (Figura 1). 
Figura 1. Pasos en la elaboración de una GPC.

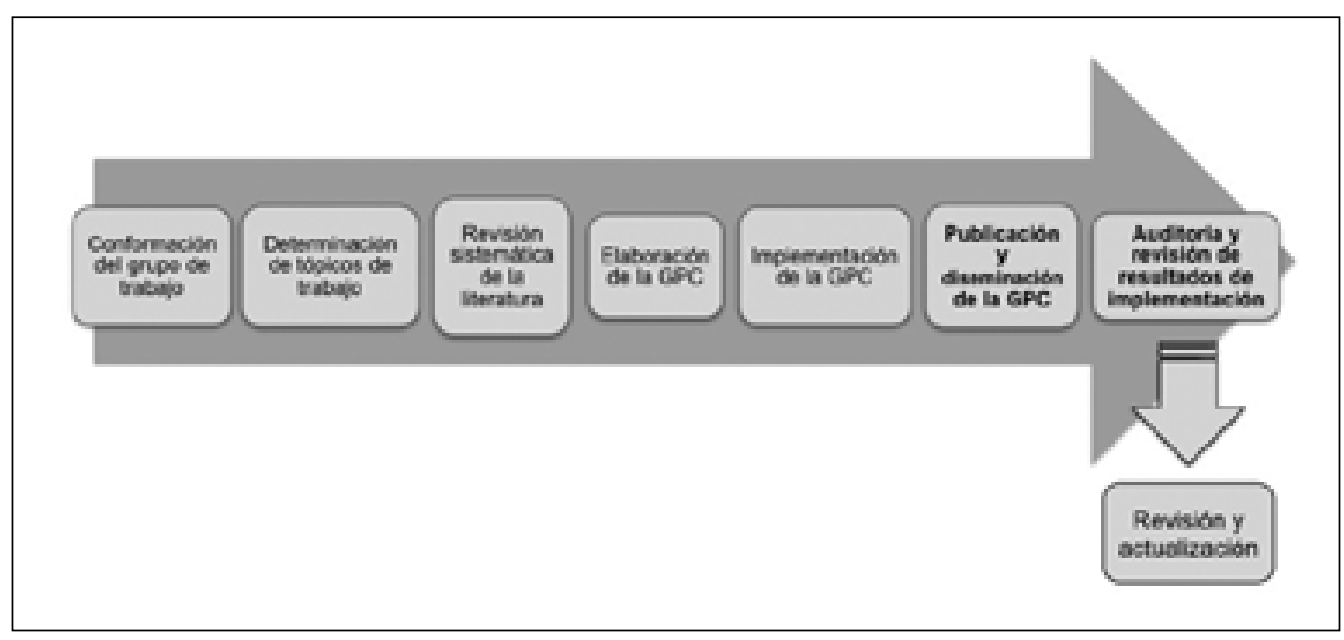

\section{Ventajas del uso de GPC basadas en la evidencia}

En primer término, las GPC permiten mejorar los resultados de salud, debido a que promueven acciones sanitarias adecuadas, reducen la variabilidad injustificada en la elección de tratamientos; proporcionan directrices basadas en los mejores resultados de la investigación biomédica; $y$, nos proveen de referencias con las que contrastar nuestros desempeños ${ }^{1}$.

Por otra parte, son herramientas de gran utilidad para quienes deben planificar y gestionar gastos en salud, debido a que permiten mejorar la eficiencia de los recursos, y controlar los costes sanitarios manteniendo la calidad asistencial ${ }^{2}$.

Las GPC constituyen una de las alternativas para la incorporación de los hallazgos de la investigación científica a la práctica clínica y, de esta manera, mejorar la calidad del cuidado sanitario. Por ende, posiblemente pueden impactar en garantizar: La eficiencia en la asignación y distribución de los recursos sanitarios; La equidad en la provisión de la asistencia sanitaria; La calidad asistencial; La idoneidad en la aplicación de procedimientos asistenciales; La evaluación de la efectividad de la asistencia sanitaria; La incorporación de nueva evidencia científica; La protección judicial; Y la educación de los usuarios (Tabla 3).

\section{¿Dónde encontrar GPC?}

En general, se puede decir que son de difusión restringida, como literatura no publicada; suelen publicarse en un formato resumido y en revistas de sociedades científicas; por lo que habitualmente no están indexadas en las bases de datos tradicionales. Por otra parte, los mecanismos de búsqueda para acceder a ellas no son muy eficientes, por ejemplo,

Tabla 3. Ventajas y desventajas de las GPC

\begin{tabular}{|ll|}
\hline Ventajas & Desventajas \\
$\begin{array}{l}\text { Aseguran un estándar de calidad asistencial } \\
\text { (ej. precisión diagnóstica, racionalizar intervenciones) }\end{array}$ & No reflejan todas las situaciones clínicas \\
$\begin{array}{l}\text { Mejoran la eficiencia (ej. reducción tiempos de diagnóstico, racionali- } \\
\text { zar distribución de recursos) }\end{array}$ & $\begin{array}{l}\text { Carencia de concordancia entre el modelo y realidad (ej. protocoliza- } \\
\text { ción de algunas patologías es complejo) }\end{array}$ \\
$\begin{array}{l}\text { Facilitan la introducción de controles de calidad (ej. favorecen registro } \\
\text { de datos, pueden ser fácilmente auditables) }\end{array}$ & $\begin{array}{l}\text { Enlentecen la actividad asistencial (ej. mayor consumo de tiempo de } \\
\text { consulta) }\end{array}$ \\
$\begin{array}{l}\text { Favorecen la investigación (ej. permiten comparar protocolos, infor- } \\
\text { matizar datos, etc.) }\end{array}$ & $\begin{array}{l}\text { Provocan problemas de actitud en los médicos } \\
\text { Facilitan la docencia (ej. estimulando la reflexión) }\end{array}$ \\
\hline
\end{tabular}


en PubMed pueden estar clasificadas indistintamente bajo alguna de las siguientes palabras claves, $D e C S$, Emtree $^{\circledR}$ o MeSH: Guidelines, Practice Guideline, Practice guidelines, Consensus development conference y Consensus; lo que impide una búsqueda rápida y eficaz ${ }^{1,17}$.

Sin embargo, es Google donde resulta más simple y eficiente la búsqueda de GPC, puesto que es el motor de búsqueda más utilizado para difundirlas (Tablas 1 y 2).

\section{¿Cómo evaluar una GPC?}

Existen instrumentos preparados para evaluar una GPC, que valoran diferentes aspectos de la estructura de esta. Uno de estos instrumentos es el AGREE II, que consta de 23 ítems organizados en 6 dominios; seguidos de 2 ítems de puntuación global (evaluación global). Cada dominio representa una dimensión única de la calidad de la GPC; y la evaluación global incluye una puntuación de la calidad general de la guía, y respecto de si la guía debe ser recomendada para su utilización en la práctica o no. Todos los ítems de AGREE II están graduados mediante una escala tipo Likert de 7 puntos $(1=$ Muy en desacuerdo, a $7=$ Muy de acuerdo $)^{18,19}$ (Tabla 4).

\section{Ejemplos}

A continuación, se resumirán algunas GPC para ejemplificarlas:

Tabla 4. Instrumento para evaluación de guías de práctica clínica. AGREE II

\section{Dominio \\ Alcance y objetivo aluden al propósito general de la guía, a los aspectos de salud específicos y a la población diana}

Participación de implicados, se refiere al grado en el que la guía fue elaborada por los interesados, y representa los puntos de vista de los usuarios a los que está destinada

Rigor en la elaboración hace referencia al proceso utilizado para reunir y sintetizar la evidencia, los métodos para formular las recomendaciones y para actualizarlas

Claridad de la presentación tiene que ver con el lenguaje, la estructura y el formato de la guía

Aplicabilidad hace referencia a las posibles barreras y factores facilitadores para su implantación, las estrategias para mejorar su adopción y las implicancias de la aplicación de la guía en los recursos

Independencia editorial tiene que ver con que la formulación de las recomendaciones no esté sesgada por conflictos de intereses

\section{Ítems}

1. ¿El objetivo general de la guía está específicamente descrito?

2. ¿El aspecto de salud cubierto por la guía está específicamente descrito?

3. ¿La población a la cual se pretende aplicar la guía está específicamente descrita?

4. ¿El grupo que desarrolla la guía incluye individuos de todos los grupos profesionales relevantes?

5. ¿Se han tenido en cuenta los puntos de vista y preferencias de la población diana?

6. ¿Los usuarios diana de la guía están claramente definidos?

7. ¿Se han utilizado métodos sistemáticos para la búsqueda de la evidencia?

8. ¿Los criterios para seleccionar la evidencia se describen con claridad?

9. ¿Las fortalezas y limitaciones del conjunto de la evidencia están claramente descritas?

10. ¿Los métodos utilizados para formular las recomendaciones están claramente descritos?

11. ¿Al formular las recomendaciones han sido considerados los beneficios en salud, los efectos secundarios y los riesgos?

12. ¿Hay una relación explícita entre cada una de las recomendaciones y las evidencias en las que se basan?

13. ¿La guía ha sido revisada por expertos externos antes de su publicación?

14. ¿Se incluye un procedimiento para actualizar la guía?

15. ¿Las recomendaciones son específicas y no ambiguas?

16. ¿Las distintas opciones para el tratamiento de la enfermedad o condición de salud se presentan claramente?

17. ¿Las recomendaciones clave son fácilmente identificables?

18. ¿La guía describe factores facilitadores y barreras para su aplicación?

19. ¿Proporciona consejo o herramientas sobre cómo las recomendaciones pueden ser llevadas a la práctica?

20. ¿Se han considerado las posibles implicancias de la aplicación de las recomendaciones sobre los recursos?

21. ¿Ofrece criterios para monitorización o auditoria?

22. ¿Los puntos de vista de la entidad financiadora no han influido en el contenido de la guía?

23. ¿Se han registrado y abordado los conflictos de interés de los miembros del grupo elaborador de la guía? 
1. GPC para el tratamiento de las infecciones intraabdominales. Sobre la base de las directrices anteriores, el equipo de trabajo detalló una serie de temas relacionados con el tratamiento de pacientes con infecciones intraabdominales $y$ luego desarrollaron preguntas clave sobre estos temas. Se realizaron búsquedas sistemáticas de la literatura, centrándose en los artículos publicados desde 2008. Estos, fueron revisados por el grupo de trabajo. Luego de obtenida la información, se generaron recomendaciones, cuya calificación se basó en el sistema GRADE, calificándose la calidad de la evidencia en alta, moderada o débil, y la fuerza de la recomendación en fuerte o débil. Se hicieron recomendaciones basadas en la evidencia respecto de riesgos en pacientes individuales, fuente de control; momento, selección y duración de la terapia antimicrobiana; y recomendaciones relacionadas con pacientes pediátricos ${ }^{20}$.

2. GPC de colecistitis aguda. Realizada con apoyo del Servicio Gallego de Salud y Fisterra. Consta de un índice de contenidos relacionados con una definición del problema, aspectos diagnósticos, terapéuticos y pronósticos; apoyados por una serie de referencias bibliográficas ${ }^{21}$.

3. GPC de hernia umbilical. Realizada con apoyo del Hospital Universitario de Getafe, Servicio Madrileño de Salud y Fisterra. Está compuesta por: definición del problema, aspectos diagnósticos, terapéuticos (indicaciones de la cirugía, opciones técnicas y sus complicaciones); apoyada por referencias bibliográficas pertinentes ${ }^{22}$.

\section{Conclusiones}

Las GPC son herramientas para mejorar el cuidado de salud a través del cambio de las conductas clínicas de los profesionales sanitarios. A pesar de su existencia por más de dos décadas, aún existen confusiones conceptuales respecto de sus características, utilidad e implementación.

La generación de este tipo de documentos es un proceso complejo, que consume recursos; en especial durante la implementación de estas, pues se requieren estrategias eficientes para conseguir los resultados esperados en los pacientes.

Las directrices futuras en el campo de la generación de GPC, se relacionan con ejecutar mejores GPC, plantear progresos de las opciones terapéuticas y realizar procesos de evaluación continua y mejora de la calidad de los procedimientos.

\section{Responsabilidades éticas}

Protección de personas y animales. Los autores declaran que para esta investigación no se han realizado experimentos en seres humanos ni en animales.

Confidencialidad de los datos. Los autores declaran que en este artículo no aparecen datos de pacientes.

Conflictos de interés: no hay.

\section{Bibliografía}

1. Guíasalud.es. Guías de práctica clínica. Disponible en: https://bit.ly/2rY83lz. Visitado el 30 de noviembre de 2018.

2. Miller M, Kearney N. Guidelines for clinical practice: development, dissemination and implementation. Int J Nurs Studies. 2004;41:813-21.

3. Alonso-Coello P, Irfan A, Solà I, Gich I, Delgado-Noguera M, Rigau D, et al. The quality of clinical practice guidelines over the last two decades: A systematic review of guideline appraisal studies. Qual Saf Heal Care 2010;19:1-8.

4. Esteve. Diario Médico. Protocolos y Guías de Práctica Clínica. Disponible en: https://bit.ly/2SfKC2u. Visitado el 28 de noviembre de 2018.
5. Shekelle PG, Woolf SH, Eccles M, Grimshaw J. Clinical guidelines: Developing guidelines. BMJ. 1999;318:593-6.

6. Shaneyfelt TM, Mayo-Smith MF, Rothwangl J. Are Guidelines Following Guidelines? JAMA 1999;281:1900.

7. Woolf SH. Evidence-based medicine and practice guidelines: An overview. Cancer Control. 2000;7:362-7.

8. Casademont J, Conthe P, Montero M. Critical evaluation of clinical practice guidelines. Med Clin (Barc). 2008;130:376-9.

9. Román A. Clinical guidelines, clinical pathways and protocols of care. Medwave 2012;12:e5436.

10. Goumard C, Farges O, Laurent A, Cherqui D, Soubrane O, Gayet B, et al. An update on laparoscopic liver resection:
French Hepato-bilio-pancreatic Surgery Association Statement. J Chir Viscerale 2015;152:107-13.

11. Takada T, Strasberg SM, Solomkin JS, Pitt HA, Gomi H, Yoshida M, et al. TG13: Updated Tokyo Guidelines for the management of acute cholangitis and cholecystitis. J Hepatobiliary Pancreat Sci. 2013;20:1-7.

12. Mortensen K, Nilsson M, Slim K, Schäfer M, Mariette C, Braga M, et al. Consensus guidelines for enhanced recovery after gastrectomy: Enhanced Recovery after Surgery (ERAS ${ }^{\circledR}$ ) Society recommendations. Br J Surg. 2014;101:1209-29.

13. Rerknimitr R, Angsuwatcharakon $P$, Ratanachu-ek T, Khor CJL, Ponnudurai $\mathrm{R}$, Moon JH, et al. Asia-Pacific consensus recommendations for endoscopic and 
interventional management of hilar cholangiocarcinoma. J Gastroenterol Hepatol. 2013;28:593-607.

14. Cong WM, Bu H, Chen J, Dong H, Zhu YY, Feng LH, et al. Practice guidelines for the pathological diagnosis of primary liver cancer: 2015 update. World J Gastroenterol. 2016;22:9279-87.

15. Yamaguchi K, Okusaka T, Shimizu K, Furuse J, Ito Y, Hanada K, et al. EBMbased Clinical Guidelines for Pancreatic Cancer (2013) issued by the Japan Pancreas Society: a synopsis. Jpn J Clin Oncol. 2014;44:883-8.

16. Navarro Puerto MA, Ruiz Romero F, Reyes Domínguez A, Gutiérrez Ibarlucea
I, Hermosilla Gago T, Alonso Ortiz del Río C, et al. ¿Las guías que nos guían son fiables? Evaluación de las guías de práctica clínica españolas. Rev Clin Esp. 2005;205:533-40.

17. García Gutiérrez JF, Bravo Toledo R. Guías de práctica clínica en Internet. Aten Primaria. 2001;28:74-9.

18. Brouwers MC, Kho ME, Browman GP, Burgers JS, Cluzeau F, Feder G, et al. AGREE II: Advancing guideline development, reporting and evaluation in health care. J Clin Epidemiol. 2010;63:1308-11.

19. Consorcio AGREE. Instrumento AGREE II. Instrumento para la evaluación de guías de práctica clínica. 2009. Available from:
https://bit.ly/1JljztM. Consultado el $11 \mathrm{de}$ diciembre de 2018.

20. Mazuski JE, Tessier JM, May AK, Sawyer RG, Nadler EP, Rosengart MR, et al. The Surgical Infection Society Revised Guidelines on the Management of Intra-Abdominal Infection. Surg Infect. (Larchmt). 2017;18:1-76.

21. Costa Ribas C, Castiñeira Pérez C. Colecistitis aguda (2017). Disponible en: https://bit.ly/2CqQwYX. Consultado el 12 de diciembre de 2018.

22. Minguez García J, Ruiz de Adana Belbel JC (2014). Hernia inguinal. Fisterra: Disponible en: https://www.fisterra. com/guias-clinicas/hernia-umbilical. Consultado el 13 de diciembre de 2018. 